\title{
Improved diagnosis of human joint
}

\author{
Norbert Leitgeb \\ Institute of Health Care Engineering with European Notified Body of Medical Devices, Graz University of Technology, Graz, Austria \\ Email: norbert.leitgeb@,tugraz.at
}

Received 2 September 2013; revised 2 October 2013; accepted 10 October 2013

Copyright (C) 2013 Norbert Leitgeb. This is an open access article distributed under the Creative Commons Attribution License, which permits unrestricted use, distribution, and reproduction in any medium, provided the original work is properly cited.

\begin{abstract}
Today, among the general population the prevalence of joint disorders is increasing even at younger ages. Since therapeutic means are sparse, early awareness of potential health problems is the most important to timely start adequate prevention. The presented improved approach of acoustic joint assessment (ACOJA) is based on subsequently measured acoustic signals emitted by joints at two different mechanical load conditions with the same measurement setup, and analysing the related relative spectral signal changes. While this approach applies to almost any joint, this paper concentrates on demonstrating the capability of this approach at hip joints. It could be demonstrated that the ACOJA method is sensible enough to allow even quantification of age-related degradations at hip normal biological human joints. It allows even identifying and accounting for gender-related differences in hip joint status. This allows concluding that ACOJA will contribute to enlarge the medical possibilities of non-invasive joint diagnostics.
\end{abstract}

Keywords: Joint Diagnosis; Ageing; Mechanical Load; Precaution

\section{INTRODUCTION}

Today, among the general population the prevalence of joint disorders is increasing even at younger ages. As an example, among the German general population $51 \%$ men and $51 \%$ women suffer from pain of muscle and joints, and $22 \%$ women and $14 \%$ men from arthrosis. Annually, about $0.5 \%$ get a joint replaced by implanted endoprostheses, among them $52.5 \%$ hip joints, $41.3 \%$ knee joints and the remainder shoulder joints, elbow joints and toe joints $[1,2]$.

Since therapeutic means are sparse, early awareness of beginning health problems is most important to allow timely prevention. However, the diagnostic tools to noninvasively assess the status of joints are limited. They comprise mainly qualitative clinical assessment and medical imaging with limited performance in this kind of diagnosis. With conventional X-ray and $\mathrm{CT}$ imaging even relevant problems with total hip implants can only be assessed with about $60 \%$ sensitivity [3]. The performance is even poorer at less severe joint disorders.

So far, approaches to enlarge the diagnostic possibilities were concentrating on hip joint implants, in particular to assess device failures or loosening by vibrometry and acoustometry. In vibrometry the response to externally provoked bone vibrations are analysed. These were generated either by sinusoidal or transient mechanical stimuli [4-7]. Vibration testing achieved $80 \%$ sensitivity and $89 \%$ specificity in identifying hip implant loosening [3]. In acoustometry the acoustic signals emitted by joints during patient's movements are picked up by sensors and analysed. In general, sensors were placed at the skin [810]. However, these approaches suffer from problems such as poor signal to noise ratio and large signal variability because of acoustic absorption by superficial tissue layers. Additional problems came from the measurement technique itself such as from sensor placement, coupling, fixation as well as sensor displacement during movement. In aiming at reducing most of these influences, the external fixation was replaced by directly embedding sensors within the femoral stem of hip implants [11]. Obviously, this limited the diagnostic application to to specific hip implants only.

This paper presents an improved acoustometric approach of acoustic joint assessment (ACOJA), suitable even for natural joints and not restricted to hip joints only. It is characterized by a high degree of standardization and improved reproducibility. In contrast to other approaches which quantitatively analyse absolute parameters of single acoustic signals, the new approach is characterized by subsequently measuring signals at two different mechanical load conditions with the same measurement setup, and analysing the relative load-induced changes of movement-induced signals. While this approach which has already been applied for patent applies 
to any joint, in this paper it is demonstrated at hip joints.

\section{METHOD}

Acoustic emissions emitted during hip joint movement were measured by a piezoelectric acceleration sensor (MT-3T, Nihon Kohden Corporation) which was placed above the frontal iliac bone and fixed by self-adhesive strips (Figure 1). Fifteen healthy volunteers were investigated ( 8 men, 7 women), ageing 21 - 64 years (men) and 21 - 58 years (women), respectively. Contraindications were acute hip problems and medication. The body mass indexes (BMI) were 20.9 - 32.5 (average 25.7) at men and 21.2 - 34.9 (average 29.1) at women, respectively. The movement activity of volunteers was assessed by a questionnaire asking for their profession and kind and average duration of their weekly movement activities such as walking, jogging, jumping or hiking both during work and spare time.

Emitted acoustic signals were measured during standardized movements such as stepping up and down a 20 $\mathrm{cm}$ step. Signals were amplified by automatic gain control and sampled with $48 \mathrm{kHz}$. Time signals were frequency-analysed and normalized to equal power. Exercises were performed without and with an additional mechanical load of $15 \mathrm{~kg}$ mass carried in a backpack. Stepping was repeated 20 times for noise reduction by post-processing.

\section{RESULTS}

The relative changes of the spectral power densities at load and no-load condition exhibited characteristic differences in terms of reduced spectral power at no-load condition in the frequency range above about $100 \mathrm{~Hz}$ and a relative increase at frequencies below (Figure 2).
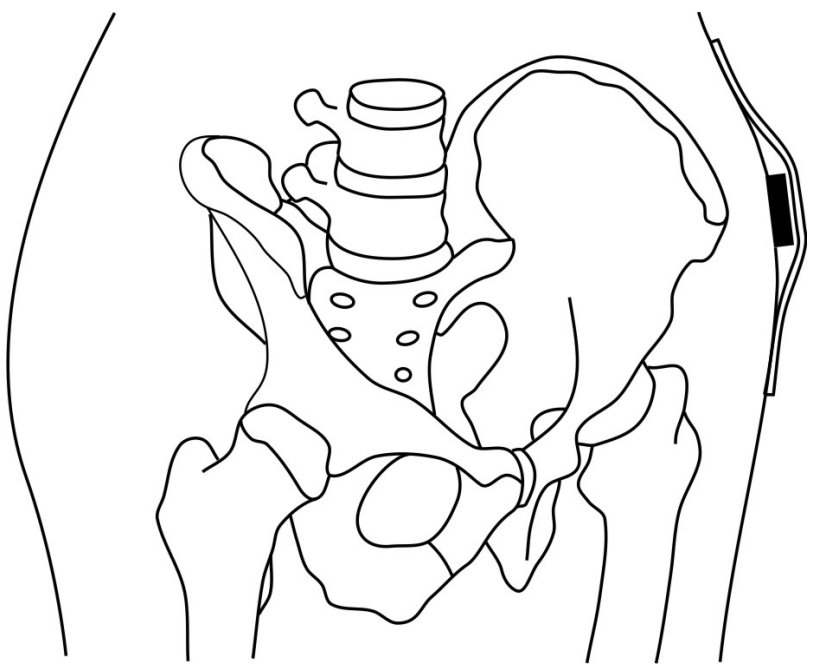

Figure 1. Location of the piezoelectric sensor above the iliac bone.
As a first sensibility check, spectral power difference should be associated with hip status in particular in dependence on age. In spite of the fact that hip joint abrasion also depends on other influences, an age-dependence could be found. Data showed gender-specific results in terms of similar dependence but shifted amplitudes. Correlation with age was moderate $(\mathrm{r}=-0.42)$, both for men and women (Figure 3).

Since higher body weight could cause increased chronic hip joint load, it was investigated whether this is reflected in the data. In fact, a better correlation of spectral power difference and body weight was found, which was strong $(\mathrm{r}=-0.60)$ for men but only weak $(\mathrm{r}=-0.37)$ for women. This is of particular importance since there was no relevant age-dependence on body weight among the group of the adult volunteers, men as well as women (Figure 4). This is in compliance with data of the general Austrian population assessed in a former study [12] comprising 501 male and 551 female ageing 10 - 82 years (Figure 5).

In addition to weight, obviously, movement activity should also play a role in joint abrasion. Therefore, due to different hip joint abrasion the data should exhibit an

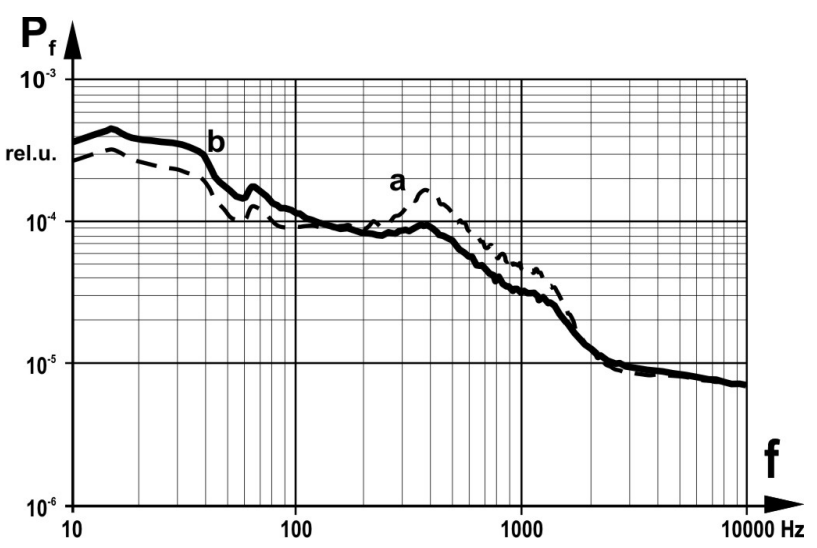

Figure 2. Spectra of power density $\mathrm{P}_{\mathrm{f}}$ of averaged over 20 steps without (a) and with $15 \mathrm{~kg}$ load (b).

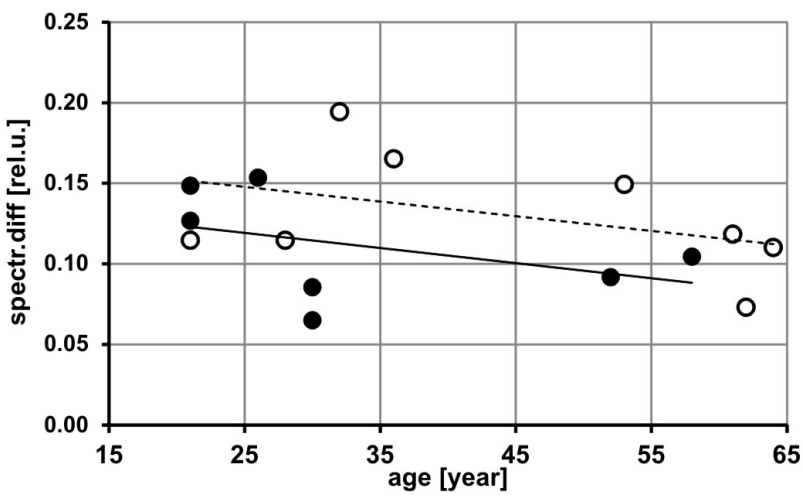

Figure 3. Spectral power differences within the frequency range $100 \mathrm{~Hz}-1000 \mathrm{~Hz}$ in dependence on age of female (full circles) male volunteers (open circles). 


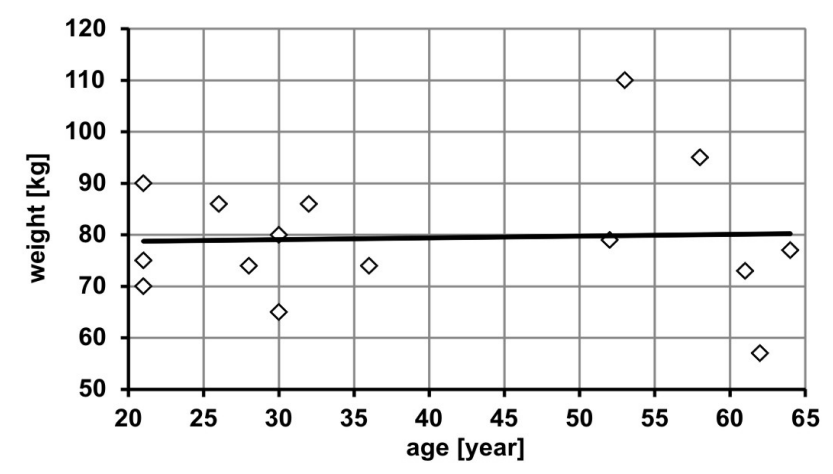

Figure 4. Body weight over age of 15 volunteers.
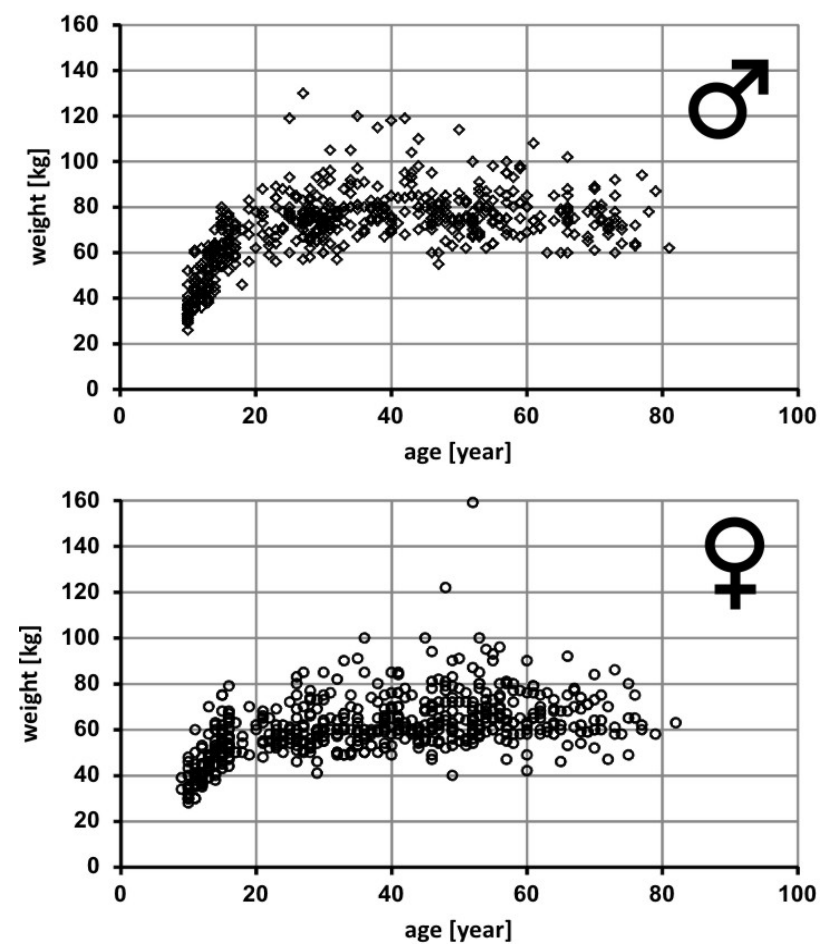

Figure 5. Body weight over age of the Austrian population, 506 men (above) and 551 women (below).

association also with mechanical activity in daily life. As expected, this was confirmed by data analysis of the weekly movement activity and of the estimated lifetime activity.

Again, the results revealed a clear gender-specific difference with almost the same dependence but shifted values (Figure 6). A moderate correlation was found at men $(\mathrm{r}=-0.51)$ and a strong correlation at women $(\mathrm{r}=$ $-0.76)$. An even stronger correlation was found at comined evaluation of age, weight and movement activity with strong correlation at men $(0.60)$ and very strong correlation at women $(0.81)$.

\section{DISCUSSION}

So far, acoustometric approaches were based on analys-

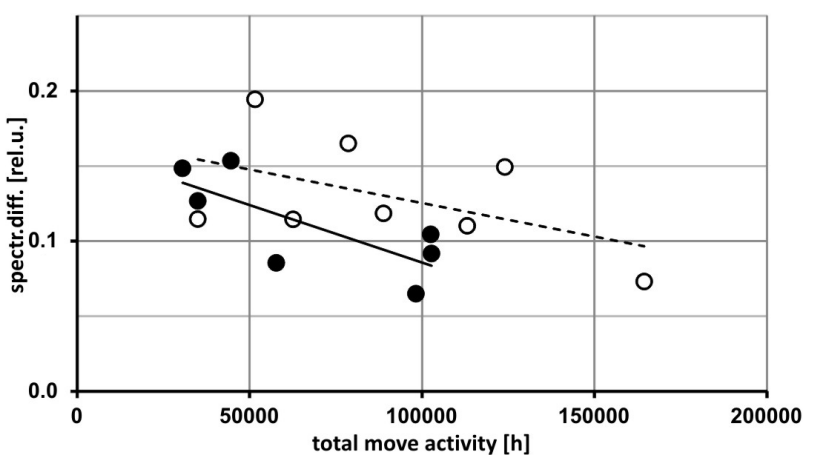

Figure 6. Dependence of the spectral difference on lifetime movement activity of men (open circles) and women (full circles).

ing stand-alone acoustic signals generated by movements of joints, but suffer from the impact of differences in sensor positioning, fixation and displacement during movement. The results of the presented improved approach demonstrate ACOJA's improved sensibility which is even high enough to allow characterisation already of nonpathologic joint alterations associated with normal joint abrasion.

Results from hip joints exhibit gender-specific differences. This can be explained by anatomical differences. The presented results show clear associations with age, body weight and movement activity. The strong correlation with movement activity has been found in spite of data originating from inquiries which by their nature are subject to recall uncertainties. It can be expected that correlation with movement activity could be further improved if activity assessment is assisted by more reliable methods such as pedometric means.

Since the results are convincing already at normal joints, it can be expected that the load/no-load differences in the acoustic emissions are much more pronounced at joints with adverse health symptoms. This will allow quantitative diagnosis such as in arthrosis or in assessing problems with implanted artificial joints.

In this investigation mechanical load has been applied by a backpack. However, load may also be applied by lead belts in case of hip joints or by lifting weights in the diagnosis of knee, elbow or shoulder joints.

Further investigations are on the way to optimise the load mass and the number of repetitive movements. The involvement of patients with clinical symptoms and joint disorders of different severity should allow generating the quantitative basis for defining diagnostic decision thresholds for adequate therapeutic intervention.

\section{CONCLUSION}

The improved acoustometric approach of analysing spectral differences of acoustic signals at two different mechanical load conditions (ACOJA) is a promising and 
cost-effective tool for non-invasive diagnosis of joints, artificial as well as biological. It could be demonstrated that ACOJA is sensible enough for quantification already of age-related degradations of normal hip joints. It allows even identifying and accounting for gender-related differences in hip joint status. This allows concluding that ACOJA will contribute to enlarge the medical possibilities of non-invasive joint diagnostics.

\section{REFERENCES}

[1] (2013) TNS Healthcare. http://de.statista.com/statistik/

[2] (2013) Welt kompakt. http://gesundheit-zahlen-daten-fakten.blogspot.co.at/2011 $\underline{12 /}$

[3] Georgiou, A.P. and Cunningham, J.L. (2001) Accurate diagnosis of hip prosthesis loosening using a vibrational technique. Clinical Biomechanics, 16, 315-323. http://dx.doi.org/10.1016/S0268-0033(01)00002-X

[4] Rowlands, A., Duck, F.A. and Cunningham, J.L. (2008) Bone vibration measurement using ultrasound: Application to detection of hip prosthesis loosening. Medical Engineering \& Physics, 30, 278-284. http://dx.doi.org/10.1016/j.medengphy.2007.04.017

[5] Glaser, D., Komistek, R.D., Cates, H.E. and Mahfouz, M.R. (2010) A non-invasive acoustic and vibration analysis technique for evaluation of hip joint condition. Biomechanics, 43, 426-432.
[6] Kohn, D.H. (1995) Acoustic emission and nondestructive evaluation of biomaterials and tissues. Critical Reviews in Biomedical Engineering, 23, 221-306. http://dx.doi.org/10.1615/CritRevBiomedEng.v23.i3-4.20

[7] Kummer, F. and Jaffe W.L. (2010) Feasibility of using ultrasonic emission for clinical evaluation of prosthetic hips. Bulletin of the NYU Hospital for Joint Diseases, 68, 262-265.

[8] Kernohan, W.G., Trainor, B., Nugent, G., Walker, P., Timoney, M. and Mollan, R. (1993) Low-frequency vibration emitted from unstable hip in human neonate. Clinical Orthopaedics and Related Research, 288, 214 218.

[9] Walter, W.L., Yeung, E. and Esposito, C. (2010) A review of squeaking hips. Journal of the American Academy of Orthopaedic Surgeons, 18, 319-326.

[10] Leitgeb, N. (1986) A new non-invasive quantitative method for fracture diagnosis. Medical Progress through Technology, 11, 185-190.

[11] Mavrogordato, M., Taylor, M., Taylor, A. and Browne, M. (2011) Real time monitoring of progressive damage during loading of a simplified total hip stem construct using embedded acoustic emission sensors. Medical Engineering \& Physics, 33, 395-406. http://dx.doi.org/10.1016/j.medengphy.2010.10.025

[12] Leitgeb, N., Schröttner, J. and Cech, R. (2007) Perception of ELF electromagnetic fields: Excitation thresholds and inter-individual variability. Health Physics, 92, 591-595. http://dx.doi.org/10.1097/01.HP.0000243128.29337.aa 